\title{
Carnets
}

Revue électronique d'études françaises de l'APEF

Première Série - 2 Numéro Spécial 10-11 | 2011

D'un Nobel l'autre

\section{Mutations du roman français depuis les années quatre-vingt : le parcours de Jean Echenoz}

Dominique Almeida Rosa de Faria

\section{OpenEdition}

1 Journals

\section{Édition électronique}

URL : http://journals.openedition.org/carnets/5362

DOI : 10.4000/carnets.5362

ISSN : 1646-7698

Éditeur

APEF

Édition imprimée

Date de publication : 1 janvier 2011

Pagination : 19-26

Référence électronique

Dominique Almeida Rosa de Faria, « Mutations du roman français depuis les années quatre-vingt : le parcours de Jean Echenoz », Carnets [En ligne], Première Série - 2 Numéro Spécial 10-11 | 2011, mis en ligne le 16 juin 2018, consulté le 21 avril 2019. URL : http://journals.openedition.org/carnets/5362 ; DOI : 10.4000/carnets.5362

\section{(c) (1) (8)}

Carnets est mis à disposition selon les termes de la licence Creative Commons - Atribution - Pas d'utilisation commerciale 4.0 International. 


\title{
MUTATIONS DU ROMAN FRANÇAIS DEPUIS LES ANNÉES QUATRE-VINGT \\ Le parcours de Jean Echenoz
}

\author{
DOMINIQUE ALMEIDA ROSA DE FARIA \\ Universidade dos Açores \\ dominiquefaria@uac.pt
}

\begin{abstract}
Résumé
Le parcours littéraire de Jean Echenoz coïncide avec les principales mutations du roman français depuis les années quatre-vingt. Lorsque cet auteur publie son premier roman, en 1979, les annonces de la mort du roman abondent dans la presse littéraire. L'aspect formel joue un rôle central dans ses premiers textes. Dans une deuxième étape de son travail (après Un An,1997), Echenoz construit dans ses ouvrages un équilibre solide entre le travail formel et le soin apporté à l'organisation de l'intrigue. C'est alors que la qualité de son travail commence à être signalée. Depuis Ravel (2006), une troisième étape commence: Echenoz participe à cette tendance au retour du sujet que l'on remarque chez un grand nombre de ses contemporains, écrivant des biographies fictionnelles de personnalités historiques. De nos jours, il est considéré comme l'un des plus importants auteurs français contemporains, et la vitalité du roman français est partout reconnue.
\end{abstract}

\begin{abstract}
If we look closely at the different stages of Jean Echenoz's work, we will find that they correspond to the most important transformations undergone by the French novel since the eighties. He published his first novel in 1979, when the literary press proclaimed the death of the novel. The formal aspects of the novel played a central role in his work then In a second stage (after Un An, 1997), this author achieves a balance between his rethinking of the formal aspects of the novel and more elaborate plots. It's then that literary critics and readers alike start noticing the quality of his work. In a third stage of his work (since Ravel (2006) Echenoz shares the tendency of the French novel nowadays to deal with biography issues. He is now considered one of the most famous French contemporary authors and the French novel is said to be alive again.
\end{abstract}

Mots-clés: Jean Echenoz, roman français contemporain, réception

Keywords: Jean Echenoz, contemporary French novel, reception 
Depuis les années cinquante, l'avis de critiques littéraires, de chercheurs et de lecteurs sur le roman français a beaucoup changé: on a commencé par annoncer sa mort et par l'accuser d'excès de formalisme, et on a fini par célébrer son renouveau, et son goût pour le récit. Ce travail se veut une réflexion sur cette mutation - celle des textes et celle de leur réception - qui s'est intensifiée après les années quatre-vingt. Mon parcours sera double: je partirai d'une réflexion sur l'œuvre d'Echenoz, qui me servira de point de départ pour saisir trois grands moments dans ce processus.

Jean Echenoz publie son premier roman en 1979. II n'est pas le seul. II appartient avec Jean-Philippe Toussaint, Eric Chevillard, Camille Laurens, Christian Oster, Antoine Volodine, Christian Gailly, entre autres - à une nouvelle génération de romanciers. L'horizon d'attente ne leur est pas favorable: aussi bien le grand public que les critiques littéraires réagissent encore au Nouveau Roman, dont les textes sont considérés hermétiques; les premières annonces de la mort du roman se font entendre.

Tous ces jeunes auteurs commencent par chercher une maison d'édition qui accepterait de publier leurs travaux. Echenoz raconte sa quête à la première personne, dans Jérôme Lindon, publié en 2001, lors du décès de ce fameux éditeur:

J'ai écrit un roman, c'est le premier, je ne sais pas que c'est le premier, je ne sais pas si j'en écrirai d'autres. Tout ce que je sais, c'est que j'en ai écrit un et que si je pouvais trouver un éditeur, ce serait bien. Si cet éditeur pouvait être Jérôme Lindon, ce serait bien sûr encore mieux, mais ne rêvons pas. Maison trop sérieuse, trop austère et rigoureuse, essence de la vertu littéraire, trop bien pour moi, même pas la peine d'essayer. J'envoie donc mon manuscrit par la poste à quelques éditeurs qui, tous, le refusent. Mais je continue, j'insiste et, au point où j'en suis, détenteur d'une collection presque exhaustive de lettres de refus, je me suis risqué la veille à déposer un exemplaire de mon manuscrit au secrétariat des Éditions de Minuit [...], sans la moindre illusion, juste pour compléter ma collection. (Echenoz, 2001: 9-10)

Certains de ces romanciers débutants ${ }^{1}$, Echenoz inclus, vont donc publier aux Editions de Minuit, ce qui n'est pas insignifiant. En effet, cette maison d'édition a, comme le montre le commentaire d'Echenoz, une réputation très bien définie de rigueur et de qualité. Bourdieu (Bourdieu, 1992: 239-240) va même plus loin et soutient qu'elle s'adresse à un public intellectuel et qu'elle représente plutôt l'avant-garde. Lorsque Jérôme Lindon décida de publier un si grand nombre de premiers romans, cela a donc attiré l'attention de la critique sur ce groupe de jeunes auteurs. S'il est vrai, toutefois, que le fait de publier aux Editions de Minuit a contribué à classer, en quelque sorte, ces romanciers, il n'est pas moins vrai que

\footnotetext{
1 Notamment, pour reprendre notre liste, Jean-Philippe Toussaint, Christian Gailly, Christian Oster et Eric Chevillard.
} 
cette nouvelle génération d'auteurs a, par la finesse de son humour et son goût du ludique, transformé l'image de sérieux généralement associée à cette maison d'édition et surpris de la sorte notamment les critiques littéraires, qui ont, à plusieurs reprises, commenté la situation inouïe ${ }^{2}$.

Ce sont aussi ces critiques littéraires qui, pour satisfaire leur besoin de classement de tout nouveau phénomène littéraire, créent la première étiquette que l'on ait essayé d'attribuer à ces auteurs - les "Nouveaux Nouveaux Romanciers". Cela montre comment le Nouveau Roman conditionne la réception de ces travaux dans les années quatre-vingt. II est vrai que cette génération a hérité de ses prédécesseurs le regard critique et méfiant par rapport aux catégories traditionnelles du roman, ainsi que le goût de l'expérimentation formelle, qu'elle développe assez pendant cette première phase de production littéraire - Echenoz affirme, dans un entretien, qu'après eux "[...] on ne pouvait plus envisager [...] l'écriture romanesque de façon naïve." (1997: 194). Ceci dit, ils articulent cette méfiance avec le goût du ludique (ils sont aussi les héritiers de Georges Perec et des membres de l'OuLiPo), avec le plaisir de raconter des histoires et, évidement, avec leurs imaginaires et leurs styles personnels. On comprend vite que cette désignation - de Nouveaux Nouveaux Romanciers - ne sert pas vraiment à les caractériser.

Cette première étape va, chez Echenoz, de 1979 à 1995 et comprend ses six premiers romans. Ceux-ci partent d'un sous-genre romanesque (le roman noir, d'aventures, d'espionnage ou policier) dont il s'amuse, non pas à détruire, mais à déjouer les règles. Dans ces premiers projets, c'est l'aspect formel qui détermine la construction du roman. L'attitude envers les traditions et les conventions littéraires est néanmoins beaucoup plus ludique que celle des nouveaux romanciers. Aussi, Echenoz, comme tous ces jeunes écrivains, reprend le goût du récit et nous trouvons dans ces romans une histoire cohérente, des péripéties, des personnages bien conçus et à l'identité stable: de quoi séduire le lecteur et l'inciter à poursuivre sa lecture. Petit à petit, le plaisir de lire redevient le but du romancier, sans que cela implique une conception naïve de la fiction.

Au long de ces années, Echenoz reçoit le prix Fénéon pour son premier roman Le méridien de Greenwich, en 1979, puis le prix Médicis pour Cherokee, en 1983, ce qui attire l'attention des critiques. Après le manque de succès de la dénomination "Nouveaux Nouveaux romanciers", l'on continue à vouloir créer un nouveau groupe d'auteurs, et, pour mieux les classer, on crée de nouvelles désignations. Ainsi, le directeur des Éditions de Minuit, désireux d'exploiter l'intérêt porté à ces jeunes romanciers, crée l'expression

\footnotetext{
2 "Les Editions de Minuit autrefois championnes du nouveau roman donnent l'impression de l'austérité, de la recherche de l'expérience. Jean Echenoz s'inscrit en faux contre cette image. II a le verbe précis et le don si rare de l'humour." (Cusin, P., 3 novembre 1999: 34). "Dire d'emblée d'un livre présenté sous le label "Éditions de Minuit" qu'il est drôle et même très drôle, cela peut passer pour de la provocation." (NURIDSANY, M., 3 septembre 1983: 26).
} 
"romanciers impassibles"3. Plus tard, ils seront plutôt appelés tout simplement "Les écrivains de Minuit" ${ }^{4}$. Ceci dit, le commentaire suivant, paru dans Libération, lors de la publication de L'équipée malaise en 1987 caractérise bien la situation:

Ils ne portent pas d'uniforme, se déplacent sans bannière [...] incognito. Ils n'ont pas derrière eux une revue, et se méfient des déclarations. Age moyen: 30-40 ans. Peu de livres encore à leur actif: ils ont pris leur temps. Ils sont discrets, épars, inclassables, prêts à déjouer tout système d'étiquetage. (Alphant, 1987: 33)

En effet, bien que l'on ait tendance à identifier les caractéristiques que ces auteurs ont en commun, on va vite comprendre qu'ils refusent d'être transformés en un groupe. La multiplicité des dénominations citées ci-dessus - notons qu'aucune n'a vraiment été adoptée - nous semble montrer à la fois le besoin de cataloguer et de former des groupes littéraires et le désarroi de la critique devant ces nouvelles formes romanesques, si difficiles à classer.

La deuxième étape du travail d'Echenoz commence avec l'écriture d'Un An en 1997. Pendant cette phase, qui comprend la publication de Au piano en 2003, Echenoz construit dans ses ouvrages un équilibre plus solide entre le travail formel et le soin apporté à l'organisation de l'intrigue. En effet, les intrigues y sont plus soignées et l'approche de l'aspect formel devient plus subtile et plus complexe: les procédés (pastiches, citations cachées, renvois intratextuels, commentaires autoriels) envahissent le roman, mais y construisent une sorte de réseau interprétatif sous-terrain, tandis que la musicalité du style cache le travail qui le soutient.

C'est pendant cette période que ces romanciers se font connaître. La critique signale chaque publication d'un nouveau roman, faisant, à chaque fois, l'éloge de leur virtuosité stylistique. En effet, c'est leur capacité à créer de l'intérêt et du plaisir à partir des jeux formels qui semble étonner le plus. Pour ce qui est d'Echenoz, il reçoit le prix Gouncourt pour Je m'en vais, en 1999 et ses ouvrages commencent à être étudiés d'abord au lycée, puis à l'université, et ils sont traduits en une trentaine de langues. Les études sur le roman français du vingtième siècle, parues à la fin des années quatre-vingt-dix, consacrent des sections considérables à son œuvre et attestent définitivement de sa qualité, comme de celle des autres romanciers. On continue cependant à avoir du mal à classer le travail de ces auteurs, notamment celui d'Echenoz. Ainsi, lorsque Rabaté (1998: 112) publie, en 1998, son

\footnotetext{
3 Jérôme Lindon fait, en effet, paraître une annonce publicitaire présentant des romans de certains de ces auteurs, sous l'appellation de "romans impassibles" (La Quinzaine Littéraire, no 532, du 16 au 31 mai 1989: 9.) II expliquera plus tard, à la suite de la polémique suscitée par cette expression, qu'il entendait par "impassible" non l'absence de sentiments, mais plutôt un certain détachement, une réserve dans l'expression de ces sentiments. A noter que cette image de l'auteur "dégagé" que Lindon a voulu associer à cette nouvelle génération de romanciers correspond à la conception de l'écrivain contemporain créée, comme le montre Anne Simonin (1994: 471), par cette maison d'édition, dès les années cinquante.

${ }^{4}$ Une des premières études publiées sur ces auteurs a d'ailleurs précisément le titre Jeunes auteurs de Minuit.
} 
essai, dans la collection "Que sais-je?", sur le roman du vingtième siècle, il l'intègre dans une partie de son travail qu'il nomme "formalisme et invention"; tant Flieder (1998: 8) que Viart (1999: 139), le classent dans la partie qu'ils consacrent aux romans "Minimalistes"; tandis que Blanckeman (2002: 65), considère que ces travaux sont des "fictions joueuses". Dans l'ensemble, les caractéristiques mises en valeur dans ces sections sont le goût du romanesque, qui coexiste avec une conscience critique des conventions littéraires, l'omniprésence du ludique, le recours fréquent à la parodie, ainsi qu'une certaine contention et un goût de l'expérimentation formelle, souvent associés au minimalisme. Ce sont donc des aspects qui relèvent tous de l'adoption d'une double position, à la fois de reprise de la tradition littéraire et de distanciation critique par rapport à elle.

La dernière étape du travail d'Echenoz subit l'influence du goût du biographique, qui a mené un grand nombre de romanciers français à se consacrer, depuis quelques années déjà, à ce nouveau sous-genre hybride, la fiction biographique. Echenoz, qui n'aime guère parler de lui, n'a pas adhéré à l'autofiction. II s'est cependant lancé un nouveau défi pour stimuler sa créativité. Ainsi, il écrit, depuis 2006, des romans biographiques. Une comparaison entre les deux livres que l'auteur a déjà publiés permet d'identifier des traits communs qui semblent fonctionner comme des règles que l'auteur s'est imposées.

Ainsi, le point de départ y est le choix d'une personnalité ayant vécu pendant le vingtième siècle et dont les exploits sont connus. Echenoz en fait le récit d'une étape de sa vie: dans le premier, il raconte les dernières années de la vie du fameux compositeur Ravel, dans le second il fait le récit des années de gloire d'Emil Zatopek, un fameux coureur de fond tchécoslovaque. Ses commentaires lors d'entretiens montrent qu'il a suivi la même méthode dans les deux romans: après avoir fait une recherche approfondie sur la période historique dans laquelle l'action aura lieu et sur la vie des personnalités, il s'est imposé des contraintes. Celles-ci consistent dans l'interdiction de changer les faits historiques, de prêter au personnage des propos qu'il n'a pas eu et de décrire sa psychologie. Cette rigueur contraste avec les libertés de romancier qu'il a gardées, puisqu'il s'agit bien d'une fiction. Un des aspects les plus intéressants de ces romans est donc ce caractère double qui rend difficile de décerner ce qui y appartient à la fiction et ce qui y relève du réel. L'entre-deux ayant toujours été bien au goût de cet auteur, il est évident qu'il joue sur cette duplicité pour attirer son lecteur.

Echenoz semble aussi s'être imposé une contrainte spécifique pour chacun de ces romans. En effet, lors de la sortie de Ravel, on a beaucoup critiqué le manque de références explicites à la musique du compositeur (fait d'autant plus étrange que la musique prend toujours une place importante dans les romans de cet auteur, où les références au monde de la musique sont fréquentes). De même, Echenoz a évité de fournir le temps des courses d'Emil Zapotek, un fait essentiel lorsque l'on veut faire le récit des exploits d'un coureur. Or, 
l'auteur comble ce vide qu'il a créé, en y soignant encore plus son écriture pour reproduire, à partir de son style, les rythmes de la musique de Ravel et ceux de la course d'Emile.

Ces deux romans biographiques reprennent aussi des traits typiques du travail précédent d'Echenoz. En effet, si nous y regardons de plus près, les héros y sont toujours des hommes, des êtres solitaires, qui voyagent beaucoup, sans que rien ne change vraiment dans leur vie. Nous y trouvons aussi, bien qu'utilisés de façon discrète, des procédés ludiques et formels. Ainsi, dans ses romans précédents, l'auteur avait l'habitude d'introduire dans son texte des citations cachées d'auteurs connus, qu'il dénonçait ensuite dans ses entretiens, invitant de la sorte le lecteur à les découvrir dans une attitude nettement ludique. Or, dans Ravel, Echenoz crée un jeu semblable avec l'usage qu'il y fait de la photographie. En effet, bien que le procédé n'ait pas encore été mentionné dans des entretiens, une lecture plus approfondie du texte nous permet d'y repérer des descriptions de photographies de Ravel (Cf. pp. 70-71; 81; 92).

Aucune indication ne signale le statut différent de ces passages, sauf le caractère un peu statique et détaillé de la description. Ainsi, les lecteurs qui, comme nous, feront suivre leur lecture d'une petite recherche pour satisfaire leur curiosité et confirmer leur suspicion, découvriront avec plaisir et étonnement les photos authentiques de Ravel que la description d'Echenoz permet d'identifier de façon catégorique. Pour ce qui est de son dernier roman, Courir, Echenoz y inclut des citations d'articles de journaux sur Emil Zatopek, un procédé qu'il trahit, cette fois-ci, lors d'un entretien ${ }^{5}$. Echenoz réussit ainsi à varier suffisamment ses projets pour ne pas se lasser et ne pas lasser ses lecteurs, tout en reprenant, réinventant, perfectionnant, les principaux traits de son écriture, auxquels ses lecteurs sont habitués.

Si nous nous arrêtons sur quelques expressions utilisées par les critiques littéraires pour caractériser l'auteur et son œuvre dans les articles de journaux et revues qui portent sur son dernier roman ${ }^{6}$, Courir, paru en 2008, nous constatons qu'il est envisagé comme un des auteurs français contemporains les plus connus. En effet, ses romans sont, comme nous l'avons mentionné, traduits dans près de trente langues et son œuvre est étudié au lycée et à l'université (et fait notamment l'objet de plusieurs projets de thèse). Les études critiques ${ }^{7}$

\footnotetext{
5 "A la Bibliothèque nationale, j'ai dépouillé 3000 exemplaires du quotidien L'Equipe de 1946 à 1957, des premiers entrefilets consacrés à ce coureur tchécoslovaque étonnant aux Unes où il devient une figure mythique. Je recopiais sur un cahier et, rentré chez moi, je tapais tout ça. Certains de ces articles avaient des qualités littéraires formidables. Parfois il fallait négocier avec ça, parfois j'ai réutilisé des images."

6 "Prix Médicis, prix Goncourt (entre autres), Jean Echenoz fait partie de célébrités de la littérature contemporaine." (Yves Loisel (Le Télégramme.com, 26/04/2009); "Il faut dire que Jean Echenoz, prix Goncourt, prix Médicis et l'on en passe, est l'un des écrivains français les plus connus et traduits dans le monde: l'œuvre est répandue dans une trentaine de langues [...]" (Jean-Louis Ezine (bibliobs.nouvelobs.com, 09/10/2008); "Portrait d'un écrivain en haut du podium." (Baptiste Liger (In: Lire, octobre 2008: 60)

7 Dont les plus récentes sont: Jerusalem, C., (2005). Le roman géographique de Jean Echenoz. Paris: Publications de I'Université de Saint-Etienne; Houppermans, Sjef (2008). Jean Echenoz. Paris: Bordas; Vray, J.B., Jérusalem, C. (dir.) (2006). Jean Echenoz: une tentative modeste de description du monde. Paris: Publications de l'Université de Saint-Etienne; Dytrt, Petr, (2007). Le (post)moderne des romans de Jean Echenoz: de l'anamnèse du moderne vers une écriture du postmoderne. Brno: Masarykova universita.
} 
ainsi que les colloques ${ }^{8}$ portant sur son œuvre deviennent de plus en plus nombreux et il a depuis longtemps une place assurée dans les histoires de la littérature française au XXème siècle. La qualité du travail d'Echenoz est donc reconnue aujourd'hui, en France comme ailleurs, ainsi que celle du roman français contemporain. Celui-ci a fait preuve d'une grande capacité de se métamorphoser et d'une grande vitalité.

\footnotetext{
${ }^{8}$ Deux de ces colloques ont même eu pour seul sujet l'œuvre d'Echenoz: “Jean Echenoz. Premier colloque international", tenu à l'Université Jean Monnet Saint-Etienne, en 2004 et "Jean Echenoz, Jeux de fictions et fictions de je", Les $4^{\mathrm{e}}$ rencontres de Chaminadour qui a eu lieu au Guéret, en 2009.
} 


\section{Bibliographie}

ALPHANT, M. (1987). “Lord Echenoz”. In: Libération, 8 janvier, pp.33-34.

Ammouche-Kremers, M. \& HILlenaAR, H. éd. (1994). Jeunes Auteurs de Minuit. Amsterdam, Atlanta: Rodopi.

Blanckeman, B. (2002). Les Fictions singulières. Paris: Prétexte éditeur.

BouRdieU, P. (1992). Les Règles de l'art. Paris: Seuil.

Cusin, P. (1999). “Jean Echenoz: aventures et pirouettes". In: Le Figaro, 3 novembre, p.34.

DYTRT, Petr, (2007). Le (post)moderne des romans de Jean Echenoz: de l'anamnèse du moderne vers une écriture du postmoderne. Brno: Masarykova universita.

ECHENOZ, J. (1979). Le Méridien de Greenwich. Paris: Minuit.

ECHENOZ, J. (1983). Cherokee. Paris: Minuit.

ECHENOZ, J. (1986). L'Equipée malaise. Paris: Minuit.

ECHENOZ, J. (1997a). Un An. Paris: Minuit.

ECHENOZ, J. (1997b). "Entretien avec Olivier Bessard Banquy: II se passe quelque chose avec le jazz". In: Europe, août-septembre, pp.194-202.

ECHENOZ, J. (1999). Je m'en vais. Paris: Minuit.

ECHENOZ, J. (2001). Jérôme Lindon. Paris: Minuit.

ECHENOZ, J. (2003). Au Piano. Paris: Minuit.

ECHENOZ, J. (2006). Ravel. Paris: Minuit.

ECHENOZ, J. (2008a). Courir. Paris: Minuit.

ECHENOZ, J. (2008b). "Entretien avec François Dufay: Zatopek, un saint laïque" In: L'express, 2-102008 [disponible le 11/12/2009] <URL: http://www.lexpress.fr/culture/livre/zatopek-un-saintlaique_823065.html>

EZINE, J.-L. (2008). "Echenoz, la course en tête". In Le nouvel observateur, n22292, du 9 au 17/10/2008. [disponible le 11/12/2009] <URL: http://www.lekiosque.fr/Le-Nouvel-Obs-numero2292-Le-pouvoir-intellectuel-en-France-online-191480.aspx>

FLIEDER, L. (1998). Le Roman français contemporain. Paris: Seuil.

HOUPPERMANS, Sjef (2008). Jean Echenoz. Paris: Bordas.

LIGER, B. (2008). "La course ou la vie". In: L'express.fr, 1-10-2008. [disponible le 11/12/2009] <URL: http://www.lexpress.fr/outils/imprimer.asp?id=815042\&k=3>

LOISEL, Y. (2009). "Jean Echenoz. Courir" In: Le Télégramme, 26 avril 2009. [disponible le 11/12/2009] <URL: http://www-prod.letelegramme.com/complements/2009/03/04/352671_echenoz.pdf>

NURIDSANY, M. (1983). "Un suspense allègre”. In: Le Figaro, 3 septembre, p.26.

RABATE, D. (1998). Le Roman français depuis 1900. Paris: P.U.F.

SIMONIN, A. (1994). Les Editions de Minuit 1942-1955. Le devoir d'insoumission. Paris: IMEC.

VIART, D. (1999). Le Roman français au $X X^{e}$ siècle. Paris: Hachette.

Jerusalem, C., (2005). Le roman géographique de Jean Echenoz. Paris: Publications de l'Université de Saint-Etienne.

VRAY, J.-B., JÉRUSAlem, C. (dir.) (2006). Jean Echenoz: une tentative modeste de description du monde. Paris: Publications de l'Université de Saint-Etienne. 